\title{
Instrumento para medir la percepción de la población con un programa de segregación en la fuente
}

\author{
Instrument to measure the perception of the population with a \\ segregation program at the source
}

Samuel García Tuanama ${ }^{1}$, Allison Angélica Núñez Vela ${ }^{2}$, Betsabeth Teresa Padilla Macedo ${ }^{3}$

Recibido: Noviembre 2020

Aceptado: Octubre 2021

\begin{abstract}
Resumen.- Uno de los principales problemas de los distritos a nivel del Perú es la inadecuada gestión de los residuos sólidos, a partir de eso se considera la importancia de tener el conocimiento adecuado del tipo de segregación de los residuos sólidos en los hogares, en el presente artículo se busca a realizó un instrumento de medición que sea útil para evaluar la percepción de la población hacía el programa de segregación en la fuente. El instrumento cuenta con una cantidad de 27 ítems y realizó una prueba piloto del instrumento realizándose en la ciudad de Tarapoto en la selva del Perú el año 2020, a una cantidad de 50 participantes, en la cual el promedio de la edad de los participantes fue de 23 años y la mayor cantidad de participantes fue del sexo femenino. Como conclusión del instrumento desarrollado en este presente trabajo de investigación, se puede observar que permite a la municipalidad ver sus errores y mejorar en su gestión del programa de Segregación en la fuente. Asimismo, para que este programa se desarrolle con éxito comienza desde el compromiso de la población siendo este el objetivo principal del instrumento desarrollado, examinar si de parte de la población hay una respuesta positiva en cuanto al programa.
\end{abstract}

Palabras clave: Segregación; percepción; instrumento de medición.

Summary.- One of the main problems of the districts at the level of Peru is the inadequate management of solid waste, from which the importance of having adequate knowledge of the type of segregation of solid waste in households is considered, in this article The aim is to create a measurement instrument that is useful for evaluating the perception of the population towards the source segregation program. The instrument has a quantity of 27 items and it carried out a pilot test of the instrument being carried out in the city of Tarapoto in the jungle of Peru with a quantity of 50 participants, in which the average age of the participants was 23 years and the largest number of participants was female. The survey had as conclusions that the instrument developed in this research work, we observe that allows the municipality to see its errors and improve its management of the Segregation program at the source. Likewise, for this program to be successfully developed, it starts from the commitment of the population, this being the main objective of the instrument developed, to examine whether there is a positive response from the population regarding the program.

Keywords: Segregation; perception; Measuring instrument.

\footnotetext{
1 Ingeniería Ambiental, Universidad Peruana Unión, samuel.garcia@upeu.edu.pe, ORCID iD: https://orcid.org/00000002-5410-9090

2 Ingeniería Ambiental, Universidad Peruana Unión, ORCID iD: https://orcid.org/0000-0001-8599-753X

${ }^{3}$ Magister en Educación, Universidad Peruana Unión, ORCID iD: https://orcid.org/0000-0002-3299-8202
}

Memoria Investigaciones en Ingeniería, núm. 21 (2021). pp. 2-14 https://doi.org/10.36561/ING.21.2 
1. Introducción. - La segregación en la fuente consiste en la escisión selectiva de los residuos sólidos en cada lugar de origen, en este caso practicándose en la población. Esta investigación permitirá obtener información sobre el nivel de compromiso de la población con respecto a la segregación de los residuos sólidos. Uno de los principales problemas de los distritos a nivel del Perú es la inadecuada gestión de los residuos sólidos, a partir de eso se considera la importancia de tener el conocimiento adecuado del tipo de segregación de los residuos sólidos en los hogares [1]. Esta investigación propone un instrumento para medir la percepción de la población respecto a un programa de segregación para poder evaluar la eficiencia de esta y la participación de la población. El instrumento servirá para la toma de decisiones a las municipalidades con respecto a la gestión, inculcar e incentivar nuevos valores a la población sobre los residuos sólidos y cultura ambiental.

2. Residuos Sólidos. - Según la Ley N 27314, Ley General de Residuos Sólidos, los residuos sólidos conforman una parte inservible de algún material que ha sido procesado, cualquier producto en estado líquido, sólidos o gaseoso, generado por actividades humanas en proceso de extracción, transformación y utilización, que está destinado a hacer desechado cuando carece de valor para la persona [2].

2.1. Clasificación. - Según el Decreto Legislativo 1278 de la Ley de Gestión Integral de Residuos Sólidos, se establece la siguiente clasificación de residuos sólidos, según su manejo y su gestión.

2.1.1. Según su manejo.- a) Residuos sólidos peligrosos: Se considera residuos sólidos peligrosos los que presentan las siguientes características: autocombustibilidad, corrosividad, explosividad, toxicidad, reactividad, radioactividad o patogenicidad, los envases que fueron utilizados para el almacenamiento o comercialización de productos o sustancias peligrosas y los productos usados o vencidos que puedan producir daños a la salud o al ambiente son considerados residuos peligrosos, deben ser manejados como tales, a menos que sean sometidos a un tratamiento que elimine las características de peligrosidad.[3].

b) Residuos sólidos no peligrosos: Los residuos sólidos no peligrosos son aquellos que son producidos por las personas en cualquier sector y aumento de su actividad, que no presentan riesgos en el ambiente y la salud. Los residuos son generados en instalaciones o por procesos industriales que no presentan las características de peligrosidad. [3].

2.1.2. Según su gestión. - De acuerdo con la autoridad competente para su gestión se clasifica en: a) Residuos sólidos municipales: Los residuos sólidos municipales (RSM), son conocidos también como basura, residuo o desecho y están compuestos por residuos orgánicos (excedentes de comida, alimentos, etc.), papel, cartón, madera y materiales inorgánicos como plástico, vidrio y metales. Estos residuos generalmente son provenientes de las actividades domésticas, construcciones, servicios públicos, establecimientos comerciales. [4].

b) Residuos sólidos no municipales: Según el Ministerio del Ambiente (MINAM) los residuos sólidos del ámbito no municipales son considerados los residuos hospitalarios, de actividad de construcción, actividades de producción industrial, actividades de agricultura y de instalaciones o actividades especiales, estas son reguladas por cada sector, siendo la responsabilidad del generador. Cada sector debe reportar al Ministerio del Ambiente (MINAM) a través de las declaraciones anuales del manejo de residuos sólidos de la gestión de estos [5].

2.2. Técnicas de minimización de residuos sólidos. - Se incluyen las tecnologías limpias y una serie de actuaciones inclinadas a reducir la cantidad o peligrosidad de los residuos sólidos generados, reducir la necesidad de tratamiento final y la conservación de los recursos [6].

Las técnicas se pueden emplear a cualquier ideal de material residual autónomo del medio receptor y no se basan necesariamente en tecnologías modernas o que requieran inmensos gastos. 
3. Segregación en la fuente. - Se refiere a la acción de la separación de los residuos sólidos que pueden ser reaprovechados en el domicilio, según el decreto legislativo 1278, nos dice que los generadores de residuos nos municipales se encuentran obligados a entregar los residuos segregados debidamente a los operadores de los residuos sólidos que son autorizados o a las municipalidades que presten el servicio.

Según la "Ley de segregación obligatoria de residuos sólidos en entidad" del 2018 en el artículo primero. -Segregación de residuos sólidos: Todas las entidades del poder Ejecutivo, los gobiernos regionales y locales y demás entidades del Estado, obligadamente segregan sus residuos sólidos según su naturaleza [7].

3.1. La segregación y reciclado de residuos en el Perú. - De acuerdo a la mencionado por el diario Peruano y junta las declaraciones de la Ministra del Ambiente, solo se recicla el 1.9\% de la basura que se recuperan en los programas de segregación de las municipalidades. Esta problemática tiene varias aristas, empezando desde la cultura de reciclaje de los propios ciudadanos peruanos.

3.2. Manejo de la segregación en la fuente. - El Ministerio del Ambiente (MINAM), implementó el "Programa de Segregación en la Fuente y Recolección Selectiva de Residuos Sólidos" desde el año 2011 en viviendas urbanas a nivel nacional, con la finalidad de reducir la cantidad y peligrosidad de los residuos sólidos dispuestos inadecuadamente, impulsando una cadena formal de reciclaje y generando un incremento de la conciencia ambiental en la ciudadanía. Tal ha sido el éxito del programa que ha logrado involucrar a 249 municipios distribuidos en las 25 regiones del Perú; generando una reducción significativa de la contaminación ambiental, mejorando la calidad de vida y educación ambiental de la población, así como la generación de nuevas oportunidades de empleo formal [8].

3.3. Programas de segregación en los municipios.- La Municipalidad José Luis Bustamante y Rivero el año 2014, realizó el "Programa de segregación en la fuente y recolección selectiva de residuos sólidos inorgánicos reciclables", debido, que hoy en día los residuos sólidos constituyen un tema crucial las diferente actividades humanas modernas y el consumismo han contribuido a acumular gran cantidad de residuos, cantidades que van en aumento cada año, siendo la causa principal la generación por parte de la población; otra causa importante la constituye las políticas antiguas de algunas municipalidades que no impulsan programas alternativos de Gestión de Residuos Sólidos centrados en la minimización y la segregación en la fuente. Sin embargo, podemos ver en los últimos años mayor cantidad de personas preocupadas por sus residuos sólidos, generalmente son aquellos que apoyan los programas de segregación en la fuente de las diferentes municipalidades del país [9].

La Municipalidad de Santiago de Surco el año 2013, implementó un programa llamado "EN SURCO LA BASURA SIRVE". Este programa consistió en sensibilizar con campañas puerta a puerta a los vecinos de la localidad, a quienes se les otorgaba una bolsa de color anaranjado para que acopiaran durante la semana los envases que podían reusarse, y luego el mismo Municipio recogía estas bolsas y clasificaba los materiales, para luego comercializarlos localmente [10].

La Municipalidad de los Olivos realizó la "Propuesta de Mejora para la gestión estratégica del Programa de Segregación en la Fuente y Recolección Selectiva de Residuos Sólidos Domiciliarios en el distrito de Los Olivos", a través de la tesis presentada por Renteria y Zaballos en el año 2014, de acuerdo a la investigación busca mejorar la gestión de los residuos sólidos domiciliarios a través de la aplicación de herramientas de gestión estratégica que permitirán diagnosticar, planificar y diseñar una serie de lineamientos estratégicos que formarán parte de la Propuesta de Mejora para el Programa de Segregación en la Fuente. Se ha abordado un desafío clave en el documento,

Memoria Investigaciones en Ingeniería, núm. 21 (2021). pp. 2-14

https://doi.org/10.36561/ING.21.2

ISSN 2301-1092 • ISSN (en línea) 2301-1106 
asimismo de realizar cambios estratégicos al Programa de estudio, que tendrá como estrategia principal la reducción de costos operativos del presupuesto del Programa con el fin de conseguir la sostenibilidad de este, y que impacte positivamente en el ahorro en el gasto del Servicio de Limpieza Pública de la Municipalidad Distrital de Los Olivos [1].

4. Materiales y métodos. - La metodología empleada para la elaboración del Instrumento para medir la percepción de la población con un programa de segregación en la fuente, estuvo compuesta por cuatro fases.

Fase 1. Preparación: Se define de manera clara y concisa los términos que serán utilizados en el instrumento, estos pueden ser mediante consulta a expertos y revisión bibliográfica [11]. Se define la población de interés y las características del formato con las preguntas de ¿para qué los queremos medir? y ¿en quienes lo queremos medir? posteriormente se realiza la elaboración del instrumento, en el que será definido el constructo, el propósito de la medición y la población de estudio, se realiza el primer borrador con los primeros ítems que nos permitirán someter la medición en el instrumento.

Fase 2. Validación: La validación por el juicio de expertos, se realiza al finalizar la primera redacción para que sea sometida a la opinión de expertos, ellos son personas que tienen la experiencia profesional, académica o investigativa con relación al tema a tratar en la investigación permitiendo valorar el contenido de cada uno de los ítems que fueron incluidos en el instrumento, dando certeza que el estilo de redacción es comprendida por el grupo al que está puesto el objetivo y por tanto asegura que las respuestas son válidas[12].

Fase 3. Prueba piloto: Se procedió a realizar una segunda redacción de los ítems que forman el instrumento que debe estar en concordancia con los resultados del análisis de los jueces expertos, la muestra que fue seleccionada puede ser al azar y tiene que ser parecida a las características de la población que fue seleccionada para la investigación, la población seleccionada fue de 50 participantes, personas locales de la ciudad de Tarapoto ubicado en la selva del Perú, compuesta de personas adultas hombres y mujeres, trabajadores o estudiantes, a partir de los 18 A 60 años que son capaces de tener conocimiento sobre la segregación de los residuos sólidos.

Fase 4. Validación: La validación psicométrica se establece el análisis de confiabilidad y la validez del test, para realizar la confiabilidad serán utilizados el software SPSS para el procesamiento de los datos recopilados y el análisis de Alfa de Cronbach que permite realizar y establecer la solidez interna de los ítems y cómo se comportan entre ellos[13], para realizar la prueba de validez según existen dos niveles de exploración para la validez de contenido: a nivel de la población y a nivel de expertos [14], la exploración a nivel de la población es cuando entrevistamos a los sujetos que más adelante serán objetos de evaluación; y a nivel de expertos es cuando entrevistamos a personas que no siendo investigadores conocen más que nosotros acerca del tema que deseamos medir.

5. Resultados y discusión.-. La edad promedio de los participantes fue 23 años (rango: 18 a 55) como se muestra en la figura I. Teniendo más participantes a adultos entre 20 a 23 años, que nos hace pensar que ellos son los que más se interesan sobre la segregación en el hogar. 


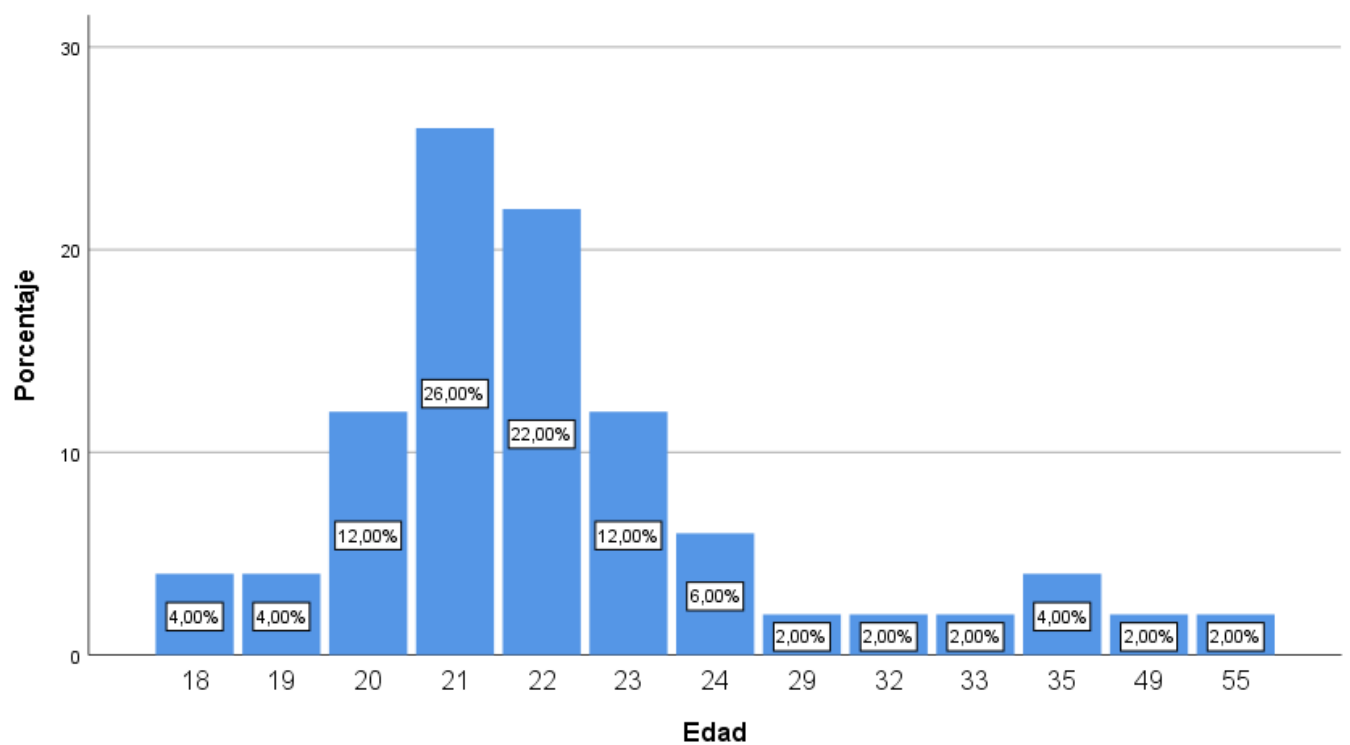

Figura I. Frecuencia de respuesta del ítem de edad de los participantes.

En la figura II se muestra la caracterización demográfica de la muestra estudiada del género en donde se obtuvo como resultado que los participantes del género femenino fueron quienes más contestaron la encuesta con un número de 32 participante el cual es el $62 \%$, dejando con el $38 \%$ de participantes del género masculino.

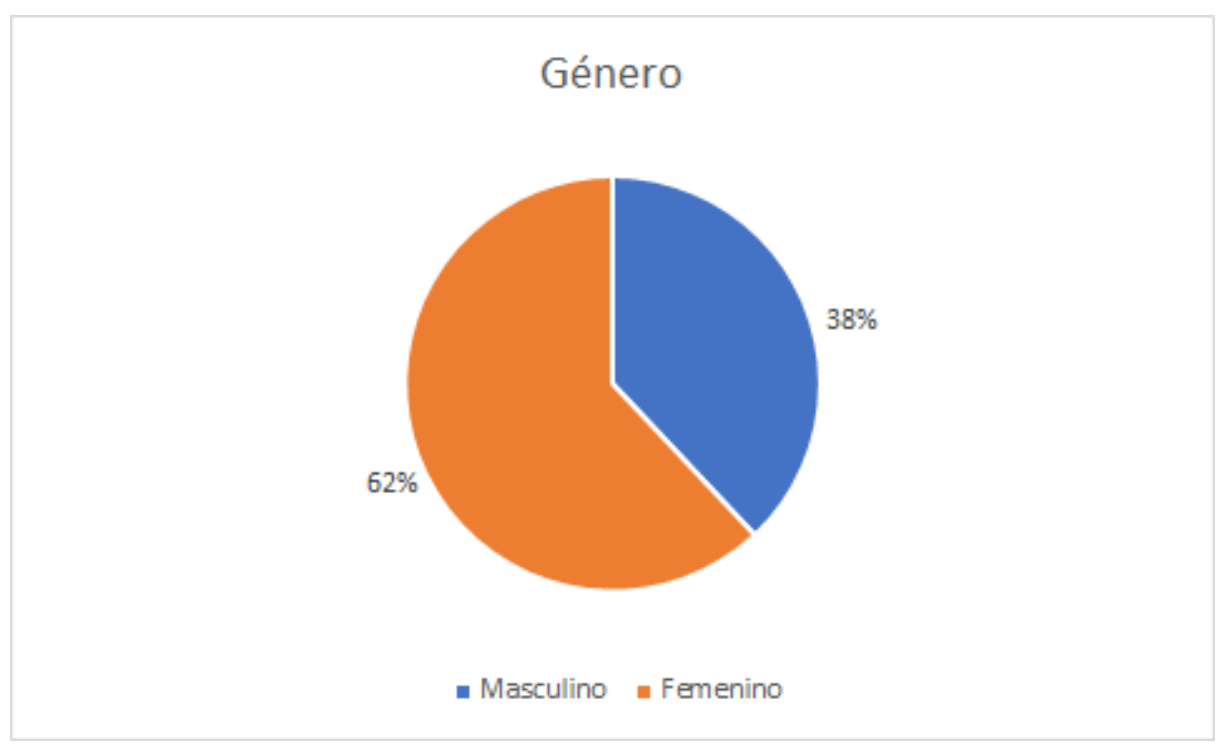

Figura II. Frecuencia de respuesta del ítem de género de los participantes.

En la figura III se muestra cuál es la dedicación de estos, en donde siendo la mayoría de los participantes estudiantes con el $44 \%$ de 22 participantes. 


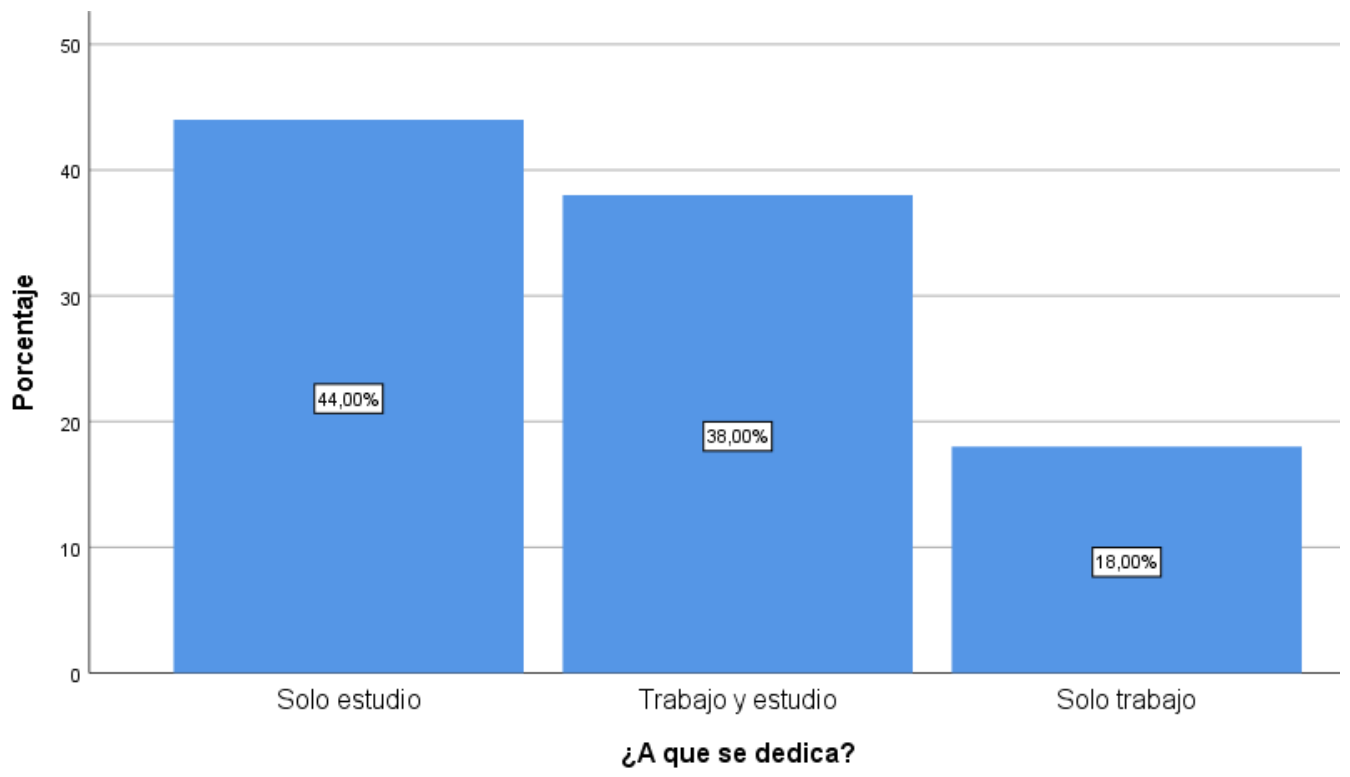

Figura III. Frecuencia de respuesta del ítem de a qué se dedican los participantes.

En la tabla I se muestra las distribuciones de frecuencia de respuesta referentes a los 27 ítems, en donde encontramos los porcentajes que se tiene de acuerdo con cada ítem con la escala propuesta.

\begin{tabular}{|c|c|c|c|c|c|c|}
\hline Ítem & $\begin{array}{l}\text { Escala de la percepción de la } \\
\text { población }\end{array}$ & Nunca & $\begin{array}{c}\text { Casi } \\
\text { nunca }\end{array}$ & Algunas veces & $\begin{array}{c}\text { Casi } \\
\text { siempre }\end{array}$ & Siempre \\
\hline \multicolumn{7}{|c|}{ ETAPA DE SENSIBILIZACIÓN } \\
\hline 1 & $\begin{array}{l}\text { ¿A menudo se realizan este } \\
\text { tipo de encuesta? }\end{array}$ & $6(12 \%)$ & $12(24 \%)$ & $25(50 \%)$ & $2(4 \%)$ & $5(10 \%)$ \\
\hline 2 & $\begin{array}{l}\text { ¿Ha menudo reciben charla } \\
\text { acerca de que son los residuos } \\
\text { sólidos? }\end{array}$ & $8(16 \%)$ & $16(32 \%)$ & $16(32 \%)$ & $5(10 \%)$ & $5(10 \%)$ \\
\hline 3 & $\begin{array}{lr}\text { ¿Sabe } \quad \text { usted } & \text { cómo } \\
\text { separar/clasificar } & \text { sus } \\
\text { residuos? } & \end{array}$ & $1(2 \%)$ & $3(6 \%)$ & $10(20 \%)$ & $16(32 \%)$ & $20(40 \%)$ \\
\hline 4 & $\begin{array}{l}\text { ¿Usted participa } \\
\text { continuamente de alguna } \\
\text { charla sobre algún programa } \\
\text { de separación de residuos } \\
\text { sólidos? }\end{array}$ & $10(20 \%)$ & $17(34 \%)$ & $14(28 \%)$ & $6(12 \%)$ & $3(6 \%)$ \\
\hline 5 & $\begin{array}{l}\text { ¿Alguna vez se le fue } \\
\text { explicada la diferencia entre } \\
\text { residuo orgánico y residuo } \\
\text { inorgánico? }\end{array}$ & $1(2 \%)$ & $4(8 \%)$ & $10(20 \%)$ & $11(22 \%)$ & $24(48 \%)$ \\
\hline 6 & $\begin{array}{l}\text { ¿Con qué frecuencia separa } \\
\text { usted sus residuos? }\end{array}$ & $4(8 \%)$ & $10(20 \%)$ & $16(32 \%)$ & $13(26 \%)$ & $7(14 \%)$ \\
\hline 7 & $\begin{array}{l}\text { Alguna vez le explicaron, } \\
\text { ¿qué son los términos de } \\
\text { residuos orgánicos } \\
\text { inorgánicos? }\end{array}$ & $2(4 \%)$ & $7(14 \%)$ & $11(22 \%)$ & $10(20 \%)$ & $10(20 \%)$ \\
\hline
\end{tabular}

Memoria Investigaciones en Ingeniería, núm. 21 (2021). pp. 2-14

https://doi.org/10.36561/ING.21.2 


\begin{tabular}{|c|c|c|c|c|c|c|}
\hline & & $\begin{array}{l}\text { Muy en } \\
\text { Desacuer } \\
\text { do }\end{array}$ & $\begin{array}{l}\text { Algo en } \\
\text { Desacuer } \\
\text { do }\end{array}$ & $\begin{array}{c}\text { Ni de } \\
\text { Acuerdo ni en } \\
\text { Desacuerdo }\end{array}$ & $\begin{array}{l}\text { Algo de } \\
\text { Acuerdo }\end{array}$ & $\begin{array}{l}\text { Muy de } \\
\text { Acuerdo }\end{array}$ \\
\hline 8 & $\begin{array}{l}\text { ¿Cree usted que separar los } \\
\text { residuos continuamente } \\
\text { puede tener un impacto } \\
\text { positivo en la salud y el } \\
\text { ambiente? }\end{array}$ & $0(0 \%)$ & $2(4 \%)$ & $6(12 \%)$ & $42(84 \%)$ & $10(20 \%)$ \\
\hline 9 & $\begin{array}{l}\text { ¿Cree usted que mediante la } \\
\text { capacitación continua } \\
\text { (charlas) se puede mejorar la } \\
\text { buena disposición de los } \\
\text { residuos sólidos? }\end{array}$ & $0(0 \%)$ & $0(0 \%)$ & $6(12 \%)$ & $14(28 \%)$ & $30(60 \%)$ \\
\hline 10 & $\begin{array}{l}\text { ¿Considera usted que realiza } \\
\text { una buena práctica con el } \\
\text { manejo de sus residuos? }\end{array}$ & $1(2 \%)$ & $6(12 \%)$ & $18(36 \%)$ & $15(30 \%)$ & $10(20 \%)$ \\
\hline \multicolumn{7}{|c|}{ ETAPA DE SEGREGACIÓN } \\
\hline 11 & $\begin{array}{l}\text { ¿Considera usted tener un } \\
\text { lugar establecido para sus } \\
\text { residuos sólidos? }\end{array}$ & $3(6 \%)$ & $5(10 \%)$ & $13(26 \%)$ & $14(28 \%)$ & $15(30 \%)$ \\
\hline 12 & $\begin{array}{l}\text { ¿Considera usted que los } \\
\text { residuos orgánicos y los } \\
\text { residuos inorgánicos deben } \\
\text { ser separados? }\end{array}$ & $0(0 \%)$ & $0(0 \%)$ & $2(4 \%)$ & $10(20 \%)$ & $38(76 \%)$ \\
\hline 13 & $\begin{array}{l}\text { ¿Considera usted importante } \\
\text { tener un lugar establecido } \\
\text { para los residuos orgánicos? } \\
\text { (cáscara de frutas y verduras, } \\
\text { papeles, restos de comida, } \\
\text { etc.) }\end{array}$ & $0(0 \%)$ & $0(\%)$ & $2(4 \%)$ & $11(22 \%)$ & $37(74 \%)$ \\
\hline 14 & $\begin{array}{l}\text { ¿Considera usted importante } \\
\text { tener un lugar establecido } \\
\text { para los residuos } \\
\text { inorgánicos? (plástico, metal, } \\
\text { etc.) }\end{array}$ & $0(\%)$ & $0(0 \%)$ & $1(2 \%)$ & $13(26 \%)$ & $36(72 \%)$ \\
\hline 15 & $\begin{array}{l}\text { ¿Considera importante tener } \\
\text { diferentes recipientes para } \\
\text { cada tipo de residuo orgánico } \\
\text { e inorgánico antes } \\
\text { mencionados? }\end{array}$ & $0(0 \%)$ & $0(0 \%)$ & $4(8 \%)$ & $11(22 \%)$ & $35(70 \%)$ \\
\hline 16 & $\begin{array}{l}\text { ¿Usted considera que al no } \\
\text { separar sus residuos, causan } \\
\text { un impacto negativo en el } \\
\text { medio ambiente? }\end{array}$ & $0(0 \%)$ & $1(2 \%)$ & $6(12 \%)$ & $15(30 \%)$ & $28(56 \%)$ \\
\hline 17 & $\begin{array}{l}\text { ¿Considera usted que sus } \\
\text { vecinos practican a menudo } \\
\text { la separación de sus residuos } \\
\text { en sus hogares } \\
\text { (Segregación)? }\end{array}$ & $13(26 \%)$ & $14(28 \%)$ & $17(34 \%)$ & $5(10 \%)$ & $1(2 \%)$ \\
\hline 18 & $\begin{array}{l}\text { ¿Considera usted que las } \\
\text { personas que viven en su } \\
\text { hogar, saben separar } \\
\text { debidamente sus residuos? }\end{array}$ & $4(8 \%)$ & $8(16 \%)$ & $16(32 \%)$ & $13(26 \%)$ & $9(18 \%)$ \\
\hline
\end{tabular}

Memoria Investigaciones en Ingeniería, núm. 21 (2021). pp. 2-14 https://doi.org/10.36561/ING.21.2 


\begin{tabular}{|c|c|c|c|c|c|c|}
\hline \multicolumn{7}{|c|}{ ETAPA DE REAPROVECHAMIENTO } \\
\hline 19 & $\begin{array}{l}\text { ¿Considera usted que es } \\
\text { importante darle otro uso } \\
\text { (reaprovechar) a su residuo? }\end{array}$ & $0(0 \%)$ & $0(0 \%)$ & $3(6 \%)$ & $12(24 \%)$ & $35(70 \%)$ \\
\hline 20 & $\begin{array}{l}\text { ¿Considera usted que al darle } \\
\text { otro uso (reaprovechar) a sus } \\
\text { residuos representan una } \\
\text { nueva oportunidad y fuente } \\
\text { de recursos? }\end{array}$ & $0(0 \%)$ & $0(0 \%)$ & $3(6 \%)$ & $10(20 \%)$ & $37(74 \%)$ \\
\hline 21 & $\begin{array}{l}\text { ¿Considera usted que está } \\
\text { haciendo reaprovechamiento } \\
\text { de sus residuos sólidos } \\
\text { orgánicos al darle las sobras } \\
\text { de comida a los animales? }\end{array}$ & $3(6 \%)$ & $4(8 \%)$ & $16(32 \%)$ & $15(30 \%)$ & $12(24 \%)$ \\
\hline & & Nunca & $\begin{array}{l}\text { Casi } \\
\text { nunca }\end{array}$ & Algunas veces & $\begin{array}{l}\text { Casi } \\
\text { siempre }\end{array}$ & Siempre \\
\hline 22 & $\begin{array}{l}\text { ¿Usted hace algún tipo de } \\
\text { reaprovechamiento de sus } \\
\text { residuos sólidos? (por } \\
\text { ejemplo, dando otro uso a las } \\
\text { botellas de plásticos o vidrios } \\
\text { en su hogar) }\end{array}$ & $3(6 \%)$ & $2(4 \%)$ & $12(24 \%)$ & $18(36 \%)$ & $15(30 \%)$ \\
\hline 23 & $\begin{array}{l}\text { ¿En qué medida usted } \\
\text { diariamente practica el } \\
\text { reaprovechamiento de sus } \\
\text { residuos sólidos? }\end{array}$ & $3(6 \%)$ & $5(10 \%)$ & $18(36 \%)$ & $19(38 \%)$ & $5(10 \%)$ \\
\hline \multicolumn{7}{|c|}{ ETAPA DE RECOLECCIÓN } \\
\hline 24 & $\begin{array}{l}\text { ¿Con qué frecuencia son } \\
\text { recogidos sus residuos por la } \\
\text { municipalidad? }\end{array}$ & $1(2 \%)$ & $5(10 \%)$ & $12(24 \%)$ & $19(38 \%)$ & $19(38 \%)$ \\
\hline 25 & $\begin{array}{l}\text { ¿Cumple la municipalidad } \\
\text { con el tiempo que se hace la } \\
\text { recolección de sus residuos } \\
\text { sólidos? }\end{array}$ & $2(4 \%)$ & $6(12 \%)$ & $16(32 \%)$ & $17(34 \%)$ & $9(18 \%)$ \\
\hline 26 & $\begin{array}{l}\text { ¿Considera usted que la } \\
\text { municipalidad siempre está } \\
\text { comprometida en la } \\
\text { recolección a tiempo de sus } \\
\text { residuos? }\end{array}$ & $1(2 \%)$ & $5(10 \%)$ & $19(38 \%)$ & $20(40 \%)$ & $5(10 \%)$ \\
\hline 27 & $\begin{array}{l}\text { ¿Está usted satisfecho con el } \\
\text { servicio de recolección de sus } \\
\text { residuos? }\end{array}$ & $2(4 \%)$ & $5(10 \%)$ & $18(36 \%)$ & $17(34 \%)$ & $8(16 \%)$ \\
\hline
\end{tabular}

Tabla I. Frecuencia de los 27 ítems propuestos para la percepción de la población en las 50 personas encuestadas.

En la tabla II se muestra el análisis psicométrico que reveló un coeficiente Alfa de Cronbach de las dimensiones de sensibilización de 0,83 , de la etapa de segregación 0,65 , la etapa de reaprovechamiento 0,71 y por último la etapa de recolección con 0,83 , con correlaciones ítem-total $-0,11$ a 0,74 , tal como se muestra en la tabla II. Para definir si procedía un análisis factorial, primero se determinó si los ítems de la "Percepción de la población con un programa de segregación en la fuente" presentan relaciones significativas. La medida de adecuación muestral KMO fue 0,638; dados estos resultados, se procedió a realizar un análisis factorial a fin de estudiar la estructura subyacente de la "Percepción de la población con un programa de segregación en la fuente" en términos de factores o dimensiones. 


\begin{tabular}{|c|c|c|c|}
\hline Ítem & Escala de la percepción de la población & $\begin{array}{l}\text { Alfa de Cronbach } \\
\text { si se elimina ítem }\end{array}$ & $\begin{array}{c}\text { Correlación } \\
\text { ítem-total }\end{array}$ \\
\hline \multicolumn{4}{|c|}{ ETAPA DE SENSIBILIZACIÓN } \\
\hline 1 & ¿A menudo se realizan este tipo de encuestas? & 0,851 & 0,115 \\
\hline 2 & $\begin{array}{l}\text { ¿Ha menudo reciben charla acerca de que son los residuos } \\
\text { sólidos? }\end{array}$ & 0,815 & 0,496 \\
\hline 3 & ¿Sabe usted cómo separar/clasificar sus residuos? & 0,794 & 0,694 \\
\hline 4 & $\begin{array}{l}\text { ¿Usted participa continuamente de alguna charla sobre } \\
\text { algún programa de separación de residuos sólidos? }\end{array}$ & 0,816 & 0,483 \\
\hline 5 & $\begin{array}{l}\text { ¿Alguna vez se le fue explicada la diferencia entre residuo } \\
\text { orgánico y residuo inorgánico? }\end{array}$ & 0,787 & 0,744 \\
\hline 6 & ¿Con qué frecuencia separa usted sus residuos? & 0,798 & 0,645 \\
\hline 7 & $\begin{array}{l}\text { Alguna vez le explicaron, ¿qué son los términos de residuos } \\
\text { orgánicos e inorgánicos? }\end{array}$ & 0,791 & 0,695 \\
\hline 8 & $\begin{array}{l}\text { ¿Cree usted que separar los residuos continuamente puede } \\
\text { tener un impacto positivo en la salud y el ambiente? }\end{array}$ & 0,823 & 0,463 \\
\hline 9 & $\begin{array}{l}\text { ¿Cree usted que mediante la capacitación continua (charlas) } \\
\text { se puede mejorar la buena disposición de los residuos } \\
\text { sólidos? }\end{array}$ & 0,823 & 0,408 \\
\hline 10 & $\begin{array}{l}\text { ¿Considera usted que realiza una buena práctica con el } \\
\text { manejo de sus residuos? }\end{array}$ & 0,813 & 0,507 \\
\hline \multicolumn{4}{|c|}{ ETAPA DE SEGREGACIÓN } \\
\hline 11 & $\begin{array}{l}\text { ¿Considera usted tener un lugar establecido para sus } \\
\text { residuos sólidos? }\end{array}$ & 0,58 & 0,475 \\
\hline 12 & $\begin{array}{l}\text { ¿Considera usted que los residuos orgánicos y los residuos } \\
\text { inorgánicos deben ser separados? }\end{array}$ & 0,601 & 0,5 \\
\hline 13 & $\begin{array}{l}\text { ¿Considera usted importante tener un lugar establecido para } \\
\text { los residuos orgánicos? (cáscara de frutas y verduras, } \\
\text { papeles, restos de comida, etc.) }\end{array}$ & 0,615 & 0,41 \\
\hline 14 & $\begin{array}{l}\text { ¿Considera usted importante tener un lugar establecido para } \\
\text { los residuos inorgánicos? (plástico, metal, etc.) }\end{array}$ & 0,611 & 0,453 \\
\hline 15 & $\begin{array}{l}\text { ¿Considera importante tener diferentes recipientes para cada } \\
\text { tipo de residuo orgánico e inorgánico antes mencionados? }\end{array}$ & 0,591 & 0,503 \\
\hline 16 & $\begin{array}{l}\text { ¿Usted considera que, al no separar sus residuos, causan un } \\
\text { impacto negativo en el medio ambiente? }\end{array}$ & 0,584 & 0,49 \\
\hline 17 & $\begin{array}{l}\text { ¿Considera usted que sus vecinos practican a menudo la } \\
\text { separación de sus residuos en sus hogares (Segregación)? }\end{array}$ & 0,752 & $-0,112$ \\
\hline 18 & $\begin{array}{l}\text { ¿Considera usted que las personas que viven en su hogar } \\
\text { saben separar debidamente sus residuos? }\end{array}$ & 0,58 & 0,475 \\
\hline \multicolumn{4}{|c|}{ ETAPA DE REAPROVECHAMIENTO } \\
\hline 19 & $\begin{array}{l}\text { ¿Considera usted que es importante darle otro uso } \\
\text { (reaprovechar) a sus residuos? }\end{array}$ & 0,675 & 0,483 \\
\hline 20 & $\begin{array}{l}\text { ¿Considera usted que al darle otro uso (reaprovechar) a sus } \\
\text { residuos representan una nueva oportunidad y fuente de } \\
\text { recursos? }\end{array}$ & 0,676 & 0,486 \\
\hline 21 & $\begin{array}{l}\text { ¿Considera usted que está haciendo reaprovechamiento de } \\
\text { sus residuos sólidos orgánicos al darle las sobras de comida } \\
\text { a los animales? }\end{array}$ & 0,738 & 0,332 \\
\hline 22 & $\begin{array}{l}\text { ¿Usted hace algún tipo de reaprovechamiento de sus } \\
\text { residuos sólidos? (por ejemplo, dando otro uso a las botellas } \\
\text { de plásticos o vidrios en su hogar) }\end{array}$ & 0,565 & 0,67 \\
\hline 23 & $\begin{array}{l}\text { ¿En qué medida usted diariamente practica el } \\
\text { reaprovechamiento de sus residuos sólidos? }\end{array}$ & 0,639 & 0,528 \\
\hline
\end{tabular}

Memoria Investigaciones en Ingeniería, núm. 21 (2021). pp. 2-14 https://doi.org/10.36561/ING.21.2 


\begin{tabular}{llcc}
\hline \multicolumn{4}{c}{ ETAPA DE RECOLECCIÓN } \\
\hline 24 & $\begin{array}{l}\text { ¿Con qué frecuencia son recogidos sus residuos por la } \\
\text { municipalidad? }\end{array}$ & 0,789 & 0,638 \\
25 & $\begin{array}{l}\text { ¿Cumple la municipalidad con el tiempo que se hace la } \\
\text { recolección de sus residuos sólidos? }\end{array}$ & 0,767 & 0,684 \\
26 & $\begin{array}{l}\text { ¿Considera usted que la municipalidad siempre está } \\
\text { comprometida en la recolección a tiempo de sus residuos? } \\
\text { ¿Está usted satisfecho con el servicio de recolección de sus } \\
\text { residuos? }\end{array}$ & 0,794 & 0,629 \\
\hline
\end{tabular}

Tabla II. Análisis psicométrico de la percepción de población hacia un programa de segregación.

En la tabla III se muestra la matriz de baremación de las puntuaciones de las encuestas por cada dimensión, en donde se realizó de acuerdo con la escala utilizada en la encuesta, teniendo un rango de puntaje para poder realizar la clasificación de indicador que da cada participante; en donde se encuentra separada por las cuatro dimensiones realizadas.

\begin{tabular}{|c|c|c|c|c|}
\hline DIMENSIONES & $\begin{array}{l}\mathrm{N}^{\circ} \mathrm{DE} \\
\text { PREGUN } \\
\text { TAS }\end{array}$ & RANGO & PUNTAJE & OBSERVACIONES \\
\hline \multirow[t]{3}{*}{$\begin{array}{c}\text { ETAPA DE } \\
\text { SENSIBILIZACIÓN }\end{array}$} & \multirow[t]{3}{*}{10} & BAJO & $1-16$ & $\begin{array}{l}\text { Indica que la población nunca fue } \\
\text { sensibilizada antes del desarrollo del } \\
\text { programa de Segregación }\end{array}$ \\
\hline & & MEDIO & $17-34$ & $\begin{array}{l}\text { Indica que la población tiene un } \\
\text { conocimiento vago acerca del Programa } \\
\text { de segregación, asimismo, que no } \\
\text { fueron sensibilizados adecuadamente }\end{array}$ \\
\hline & & ALTO & $35-50$ & $\begin{array}{l}\text { Demuestra que la población fue } \\
\text { sensibilizada antes del desarrollo de } \\
\text { programa }\end{array}$ \\
\hline \multirow[t]{3}{*}{$\begin{array}{c}\text { ETAPA DE } \\
\text { SEGREGACIÓN }\end{array}$} & \multirow[t]{3}{*}{8} & BAJO & $1-14$ & $\begin{array}{l}\text { Indica que la población no está } \\
\text { practicando la segregación referente a } \\
\text { programa }\end{array}$ \\
\hline & & MEDIO & $15-28$ & $\begin{array}{l}\text { Indica que población no } \\
\text { practicando está } \\
\text { segregación de los residuos }\end{array}$ \\
\hline & & ALTO & $29-40$ & $\begin{array}{l}\text { Indica la adecuada segregación de parte } \\
\text { de la población, asimismo una respuesta } \\
\text { positiva de parte ellos respecto al } \\
\text { programa }\end{array}$ \\
\hline \multirow[t]{3}{*}{$\begin{array}{c}\text { ETAPA DE } \\
\text { REAPROVECHAMIE } \\
\text { NTO }\end{array}$} & \multirow[t]{3}{*}{5} & BAJO & $1-9$ & $\begin{array}{l}\text { Demuestra que la población no realiza } \\
\text { ningún o poco tipo de } \\
\text { reaprovechamiento acerca de los } \\
\text { residuos orgánicos e inorgánicos. }\end{array}$ \\
\hline & & MEDIO & $10-18$ & $\begin{array}{l}\text { Demuestra que la población realiza } \\
\text { mediamente una clase de } \\
\text { reaprovechamiento hacia sus residuos } \\
\text { orgánicos e inorgánicos, ya sea } \\
\text { reaprovechando botellas plásticas o } \\
\text { dando de comer a sus animales con las } \\
\text { sobras de comida. }\end{array}$ \\
\hline & & ALTO & $19-25$ & $\begin{array}{l}\text { La población realiza todo el tipo de } \\
\text { reaprovechamiento posible hacia sus } \\
\text { residuos sólidos. }\end{array}$ \\
\hline
\end{tabular}

Memoria Investigaciones en Ingeniería, núm. 21 (2021). pp. 2-14

https://doi.org/10.36561/ING.21.2 


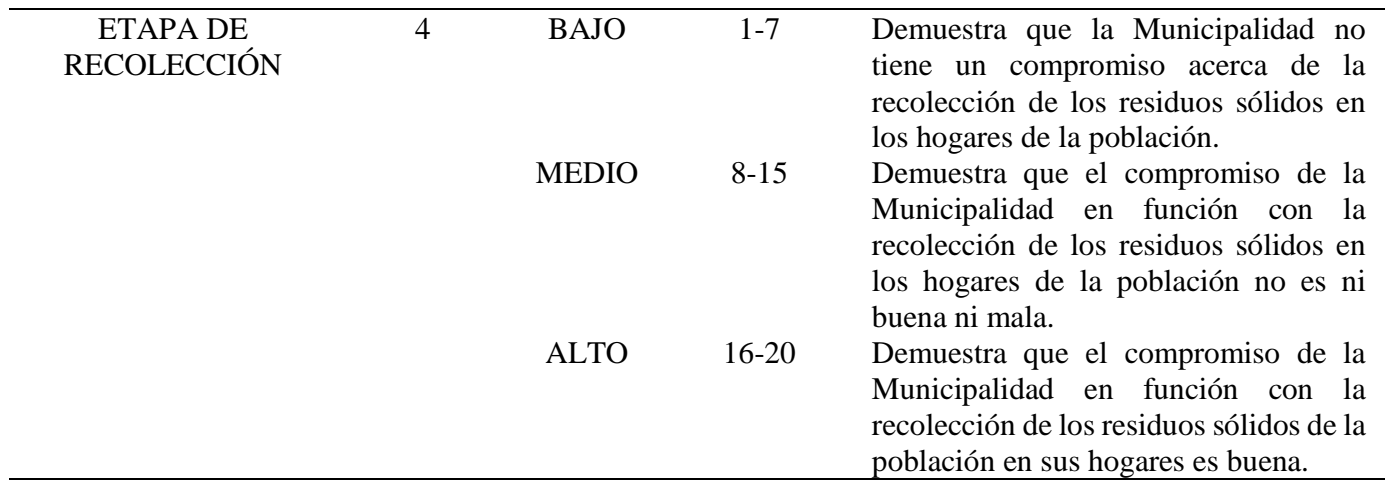

Tabla III. Baremación de las puntuaciones.

En la tabla IV se muestra el análisis de puntajes de las encuestas realizadas en donde se encuentra separada por las cuatro dimensiones realizadas mostrando el mínimo y máximo de cada dimensión, comparando los puntajes con la matriz realizada.

\begin{tabular}{lcccc}
\hline Dimensiones & $\begin{array}{c}\mathrm{N}^{\circ} \text { de } \\
\text { encuestas }\end{array}$ & Mínimo de puntaje & Máximo de puntaje & Media \\
\hline SENSIBILIZACIÓN & 50 & 20 & 47 & 35,78 \\
SEGREGACIÓN & 50 & 24 & 40 & 32,44 \\
REAPROVECHAMIENTO & 50 & 9 & 25 & 20,06 \\
RECOLECCIÓN & 50 & 6 & 20 & 14,2 \\
\hline
\end{tabular}

Tabla IV. Análisis de puntaje de encuestas realizadas.

En cuanto a los resultados obtenidos en la etapa de sensibilización con un valor máximo de 47 puntos lo cual nos muestra de acuerdo con la matriz realizada, que la población fue sensibilizada antes del desarrollo del programa, además que obtuvimos un valor mínimo de 20 puntos de acuerdo a este valor, nos indica que una parte de la población, tienen un conocimiento bajo acerca del programa, o que; de alguna manera ellos no fueron sensibilizados adecuadamente. Por otro lado, esto ocurre a menudo por la falta de compromiso de parte de la población de no querer participar, lo cual esto lleva a las municipalidades a tomar nuevas medidas en cuanto a la sensibilización hacia las personas.

Asimismo, en la etapa de segregación en la fuente el valor máximo fue de 40 puntos, de acuerdo con la matriz, indica la práctica adecuada de segregación por parte de los participantes, a su vez una respuesta positiva respecto al programa. Además, el valor mínimo fue 24 puntos de manera que esto nos indica que hay una parte de los participantes que no están practicando adecuadamente la segregación en sus hogares. Finalmente, esto llevaría hacer un seguimiento más de cerca en lo que respecta al compromiso con la segregación.

Sin embargo, en la etapa de reaprovechamiento el valor máximo fue 25 puntos a fin de que indica que la población practica todo tipo de aprovechamiento posible en sus hogares, también obtuvimos el valor mínimo de 9 puntos, nos muestra que una parte de la población no están practicando ningún tipo de aprovechamiento en sus hogares a causa de su falta de compromiso en sus residuos orgánicos e inorgánicos.

Finalmente, en la etapa de recolección el valor máximo fue de 20 de puntos en efecto, muestra evidentemente el compromiso de parte de la municipalidad en cuanto en la recolección de los 
residuos, teniendo en cuenta que el valor mínimo fue 6 puntos un indicador de la falta de compromiso de la municipalidad en algunos lugares alejados.

6. Conclusiones. - Esta investigación propuso un instrumento útil para la evaluación de la población con un programa de segregación en la fuente, en la cual los resultados nos muestran la respuesta positiva de parte de los ciudadanos en cuanto al desarrollo del programa. En la investigación se realizó el análisis psicométrico que reveló un coeficiente Alfa de Cronbach de las dimensiones en donde se tuvo en la etapa de sensibilización 0,83 , la etapa de segregación 0,65 , la etapa de reaprovechamiento 0,71 y por último la etapa de recolección con 0,83 , tal como se muestra en la tabla II para la identificación de confiabilidad de cada uno de los ítems. La medida de adecuación muestral KMO fue de 0,638; dados estos resultados, se realizó un análisis factorial a fin de estudiar la estructura subyacente de la "Percepción de la población con un programa de segregación en la fuente" en términos de factores o dimensiones. También se realizó una matriz para la baremación de las puntuaciones, para obtener mejores resultados en cada una de las dimensiones, dándoles valores de acuerdo con la escala utilizada en el instrumento.

$\mathrm{Y}$ para mejorar las respuestas negativas que se obtuvieron hacia el instrumento, este ayuda a determinar y comparar los resultados de un antes y después para mejorar el Programa de segregación en la fuente. Con el fin de alcanzar un mayor nivel de compromiso y participación hacia la población, se recomienda a la municipalidad lo siguiente: Realizar talleres de segregación buscando como meta visibilizar la importancia de un manejo adecuado de los residuos sólidos en el hogar, de esta manera generando un aporte positivo en la comunidad; y agregando a esto incentivos tributarios a los ciudadanos. Asimismo, brindando los materiales adecuados para la buena práctica del programa. 


\section{Referencias}

[1] J. M. Rentería Sacha and M. E. Zeballos Villareal, "Propuesta de Mejora para la gestión estratégica del Programa de Segregación en la Fuente y Recolección Selectiva de Residuos Sólidos Domiciliarios en el distrito de Los Olivos," 2014.

[2] Ministerio del Ambiente - MINAM, "Ley General de Residuos Sólidos," Lima, Perú, 2004. Accessed: Jun. 23, 2020. [Online].

Available: http://transparencia.mtc.gob.pe/idm_docs/normas_legales/1_0_2819.pdf

[3] Dirección de Normalización - INACAL, "Norma Técnica Peruana 900.058 - 2019 Gestión de Residuos. Códigos de colores para el almacenamiento de residuos sólidos.," no. Lima 27, pp. 114, 2019, [Online].

Available:

https://www.qhse.com.pe/wp-content/uploads/2019/03/NTP-900.058-2019-

Residuos.pdf

[4] R. Toro, M. Szantó, J. Pacheco, E. Contreras, and A. Gálvez, "Guía general para la gestión de residuos sólidos domiciliarios," Manuales la CEPAL, p. 209, 2016.

[5] Ministerio del Ambiente - MINAM, "Gestión Ambiental de Residuos Sólidos en el Perú," 2011.

[6] M. María and C. Gallo, "Minimización de Residuos: una política de gestión ambiental empresarial Waste minimization: An environmental management corporative policy," Prod. + Limpia, vol. 1, no. 2, p. 12, 2006, Accessed: Jun. 23, 2020. [Online].

Available: $\quad$ http://lasallista.edu.co/fxcul/media/pdf/RevistaLimpia/vol1n2/pl_v1n2_4657_Minimización.pdf

[7] P. CONGRESO DE LA REPÚBLICA, Ley de Segregación Obligatoria de Residuos Sólidos en Entidades Públicas. Perú, 2018, p. 8.

[8] Ministerio del Ambiente - MINAM, "Programa nacional de segregación en la fuente y recolección selectiva de residuos sólidos|Dirección General de Calidad Ambiental," 2011. http://www.minam.gob.pe/calidadambiental/programa-nacional-de-segregacion-en-la-fuente-y-

recoleccion-selectiva-de-residuos-solidos/ (accessed Oct. 29, 2019).

[9] Municipalidad José Luis Bustamante y Rivero, "Programa de segregación en la fuente y recolección selectiva de residuos sólidos inorgánicos reciclables,” 2014. Accessed: Nov. 16, 2019. [Online].

Available: http://www.munibustamante.gob.pe/archivos/gestamb/psfrsrsi2014.pdf

[10] Municipalidad de Santiago de Surco, "Premio Interamericano a la Innovación para la Gestión Pública Efectiva," p. 16, 2013, [Online]. Available: www.munisurco.gob.pe.

[11] M. C. M. Arribas, "Diseño y validación de cuestionarios Formación continuada El cuestionario es un instrumento para la recogida de información, diseñado para cuantificarla y universalizarla," 2004.

[12] A. M. Soriano Rodríguez, "Diseño y validación de instrumentos de medición," Diá-logos, no. 14, pp. 19-40, Nov. 2015, doi: 10.5377/dialogos.v0i14.2202.

[13] H. C. Oviedo and A. Campo-Arias, "Metodología de investigación y lectura crítica de estudios Aproximación al uso del coeficiente alfa de Cronbach Title: An Approach to the Use of Cronbach's Alfa," Rev. Colomb. Psiquiatr. ISSN, vol. 34, no. 4, pp. 572-580, 2005, Accessed: Jun. 23, 2020. [Online]. Available: https://www.redalyc.org/pdf/806/80634409.pdf

[14] J. Supo, Cómo validar un instrumento: La guía para validar un instrumento en 10 pasos. 2013. 